\title{
On the nonlinear dynamics of the traveling-wave solutions of the Serre system
}

\author{
Dimitrios Mitsotakis ${ }^{\mathrm{a}, *}$, Denys Dutykh ${ }^{\mathrm{b}}$, John Carter ${ }^{\mathrm{c}}$ \\ ${ }^{a}$ Victoria University of Wellington, School of Mathematics, Statistics and Operations \\ Research, PO Box 600, Wellington 6140, New Zealand \\ ${ }^{b}$ LAMA, UMR 5127 CNRS, University Savoie Mont Blanc, Campus Scientifique, 73376 \\ Le Bourget-du-Lac Cedex, France \\ ${ }^{c}$ Mathematics Department, Seattle University, 901 12th Avenue, Seattle, WA 98122, USA
}

\begin{abstract}
We numerically study nonlinear phenomena related to the dynamics of traveling wave solutions of the Serre equations including the stability, the persistence, the interactions and the breaking of solitary waves. The numerical method utilizes a high-order finite-element method with smooth, periodic splines in space and explicit Runge-Kutta methods in time. Other forms of solutions such as cnoidal waves and dispersive shock waves are also considered. The differences between solutions of the Serre equations and the Euler equations are also studied.
\end{abstract}

Keywords: Solitary waves, cnoidal waves, stability, finite element method 2000 MSC: 76B15, 76B25, 76M10

\section{Introduction}

The Serre equations (also known as the Green-Naghdi or Su-Gardner equations) $[1,2,3]$ approximate the Euler equations of water wave theory and model the one-dimensional, two-way propagation of long waves. If $a$ denotes a typical amplitude of a wave, $d$ the mean depth of the fluid, and $\lambda$ a typical wavelength, then the Serre equations are characterized by the parameters

\footnotetext{
*Corresponding author

Email addresses: dimitrios.mitsotakis@vuw.ac.nz (Dimitrios Mitsotakis), Denys.Dutykh@univ-savoie.fr (Denys Dutykh), carterj1@seattleu.edu (John Carter)
}

Preprint submitted to Wave Motion

July 13, 2016

(C) 2016. This manuscript version is made available under the Elsevier user license http://www.elsevier.com/open-access/userlicense/1.0/ 
$\varepsilon \doteq a / d=\mathcal{O}(1)$ and $\sigma \doteq d / \lambda \ll 1$, contrary to the Boussinesq equations which model the propagation of small-amplitude, long waves, i.e. $\varepsilon \ll 1$ and $\sigma \ll 1$, when the Stokes number is $S \doteq \varepsilon / \sigma^{2}=\mathcal{O}(1)$. The Boussinesq equations are often called weakly nonlinear, weakly dispersive equations while the Serre equations are often called fully-nonlinear shallow-water equations. In dimensionless and scaled variables, the Serre equations take the form:

$$
\begin{aligned}
& \eta_{t}+u_{x}+\varepsilon(\eta u)_{x}=0, \\
& u_{t}+\eta_{x}+\varepsilon u u_{x}-\frac{\sigma^{2}}{3 h}\left[h^{3}\left(u_{x t}+\varepsilon u u_{x x}-\varepsilon\left(u_{x}\right)^{2}\right)\right]_{x}=0,
\end{aligned}
$$

for $x \in \mathbb{R}, t>0$, along with the initial conditions

$$
\eta(x, 0)=\eta_{0}(x), \quad u(x, 0)=u_{0}(x) .
$$

Here $\eta=\eta(x, t)$ is the free surface displacement, while

$$
h \doteq 1+\varepsilon \eta,
$$

is the total fluid depth, $u=u(x, t)$ is the depth-averaged horizontal velocity, and $\eta_{0}, u_{0}$ are given real functions, such that $1+\varepsilon \eta_{0}=h_{0}>0$ for all $x \in \mathbb{R}$. In these variables, the location of the horizontal bottom is given by $y=-1$. For a review of the derivation and the basic properties of this system we refer to $[4]$.

The Euler equations along with the model system (1) admit traveling wave solutions, i.e. waves that propagate without change in shape or speed $[5,6,7]$. Solitary waves form a special class of traveling wave solutions of these systems. The other important class of traveling wave solutions is the class of cnoidal wave solutions which can be thought of as the periodic generalization of solitary waves. Many Boussinesq-type equations are known to possess solitary wave and periodic solutions, but do not admit nontrivial, closed-form solutions. In contrast, the Serre equations admit closed-form solitary and cnoidal (periodic) wave solutions. The solitary wave solutions of the Serre system traveling with constant speed $c_{s}$ are given by

$$
h_{s}(\xi)=\left(a_{0}+a_{1} \operatorname{sech}^{2}\left(K_{s} \xi\right)\right) / \sigma, \quad u_{s}(\xi)=c_{s}\left(1-\frac{a_{0}}{\sigma h_{s}(\xi)}\right) / \epsilon,
$$

where $\xi=x-c_{s} t, K_{s}=\sqrt{3 a_{1} / 4 \sigma a_{0}^{2} c_{s}^{2}}, c_{s}=\sqrt{\left(a_{0}+a_{1}\right) / \sigma}, a_{0}>0$, and $a_{1}>0$. By taking $a_{0}=\sigma$ and $a_{1}=\varepsilon \sigma A_{s}$ the formulas for the classical solitary waves that are homoclinic to the origin are obtained. 
The cnoidal waves of the Serre system traveling with constant speed $c_{c}$ are given by

$$
h_{c}(\xi)=\left(a_{0}+a_{1} \operatorname{dn}^{2}\left(K_{c} \xi, k\right)\right) / \sigma, \quad u_{c}(\xi)=c_{c}\left(1-\frac{h_{0}}{h(\xi)}\right) / \epsilon,
$$

where $h_{0}=a_{0}+a_{1} E(m) / K(m), K_{c}=\sqrt{3 a_{1}} / 2 \sqrt{a_{0}\left(a_{0}+a_{1}\right)\left(a_{0}+\left(1-k^{2}\right) a_{1}\right)}$, $c_{c}=\sqrt{a_{0}\left(a_{0}+a_{1}\right)\left(a_{0}+\left(1-k^{2}\right) a_{1}\right) / \sigma h_{0}^{2}}, k \in[0,1], m=k^{2}, a_{0}>0$, and $a_{1}>0$. Here $K$ and $E$ are the complete elliptic integrals of the first and second kind respectively. Note that (4) are the $k \rightarrow 1$ limit of (5).

Another fundamental property of the Serre system is the conservation of the energy which plays also the role of the Hamiltonian, $\mathcal{H}(t)=\frac{1}{2} \int_{-\infty}^{\infty}\left(\varepsilon h u^{2}+\right.$ $\left.\frac{\varepsilon \sigma^{2}}{3} h^{3} u_{x}^{2}+\varepsilon \eta^{2}\right) d x$, in the sense that $\mathcal{H}(t)=\mathcal{H}(0)$ for all $t>0$ up to the maximal time $T$ of the existence of the solution.

In this paper we study the problem of the nonlinear stability (orbital and asymptotic) of the traveling waves of the Serre system by using numerical techniques. We provide numerical evidence of stability with respect to certain classes of perturbations. Phenomena such as perturbations of the traveling waves, perturbations of the Serre system and interactions of traveling waves are studied analyzing the stability properties of the waves at hand. We also study the interactions of dispersive shock waves (DSWs) in the Serre system. The physical relevance of the Serre equations is addressed whenever possible.

The paper is organized as follows. The numerical method is presented briefly in Section 2. The compatibility of the solitary waves of the Serre and the Euler systems is examined in Section 3. The head-on collision of solitary waves is studied in Section 3.2. A number of issues related to the stability of the traveling waves are discussed in Sections 4. The interaction of DSWs is presented in Section 5 .

\section{The numerical method}

The numerical method of preference is a high-order Galerkin / Finite element method (FEM) for the spatial discretization combined with the classical fourth-order explicit Runge-Kutta method in time. In some cases adaptive time-stepping methods, such as the Runge-Kutta-Fehlberg, the Cash-Karp and the Dormand-Prince methods [8], were employed to verify that there are no spurious solutions or blow-up phenomena. This numerical scheme has been shown to be highly accurate and stable since there is no need for a 
restrictive condition on the step-size but only mild conditions of the form $\Delta t \leq C \Delta x$ cf. [9]. The conservation of the Hamiltonian was monitored and was usually conserved to within 8 to 10 significant digits. In order to ensure the accuracy of the numerical results obtained with the FEM we compared most with the analogous results obtained with the pseudo-spectral method described and analyzed in [10]. The experiments presented in this paper also serve as numerical benchmarks for the efficacy of the numerical scheme.

We consider (1) with periodic boundary conditions and, for simplicity, assume $\varepsilon=\sigma=1$. We rewrite (1) in terms of $(h, u)$ rather than $(\eta, u)$. This is done by using (3) and yields the initial-boundary value problem

$$
\begin{aligned}
& h_{t}+(h u)_{x}=0, \\
& u_{t}+h_{x}+u u_{x}-\frac{1}{3 h}\left[h^{3}\left(u_{x t}+u u_{x x}-\left(u_{x}\right)^{2}\right]_{x}=0,\right. \\
& \partial_{x}^{i} h(a, t)=\partial_{x}^{i} h(b, t), \quad i=0,1,2, \ldots, \\
& \partial_{x}^{i} u(a, t)=\partial_{x}^{i} u(b, t), \quad i=0,1,2, \ldots, \\
& h(x, 0)=h_{0}(x), \\
& u(x, 0)=u_{0}(x),
\end{aligned}
$$

where $x \in[a, b] \subset \mathbb{R}$ and $t \in[0, T]$. Considering a spatial grid $x_{i}=a+i \Delta x$, where $i=0,1, \cdots, N, \Delta x$ is the spatial mesh length, and $N \in \mathbb{N}$, such that $\Delta x=(b-a) / N$. We define the space of the periodic cubic splines

$$
S=\left\{\phi \in C_{\mathrm{per}}^{2}[a, b]|\phi|_{\left[x_{i}, x_{i+1}\right]} \in \mathbb{P}^{3}, 0 \leq i \leq N-1\right\}
$$

where $C_{\text {per }}^{2}=\left\{f \in C^{2}[a, b] \mid f^{(k)}(a)=f^{(k)}(b), 0 \leq k \leq r\right\}$ and $\mathbb{P}^{k}$ is the space of polynomials of degree $k$. The semi-discrete scheme is reduced to finding $\tilde{h}, \tilde{u} \in S$ such that

$$
\begin{aligned}
& \left(\tilde{h}_{t}, \phi\right)+\left((\tilde{h} \tilde{u})_{x}, \phi\right)=0, \\
& \left.\mathcal{B}\left(\tilde{u}_{t}, \phi ; \tilde{h}\right)+\left(\tilde{h}\left(\tilde{h}_{x}+\tilde{u} \tilde{u}_{x}\right), \phi\right)+\frac{1}{3}\left(\tilde{h}^{3}\left(\tilde{u} \tilde{u}_{x x}-\left(\tilde{u}_{x}\right)^{2}\right), \phi_{x}\right)\right)=0,
\end{aligned}
$$

where $\mathcal{B}$ is defined as the bilinear form that for fixed $\tilde{h}$ is given by

$$
\mathcal{B}(\psi, \phi ; \tilde{h}) \doteq(\tilde{h} \psi, \phi)+\frac{1}{3}\left(\tilde{h}^{3} \psi_{x}, \phi_{x}\right) \text { for } \phi, \psi \in S .
$$

The system of equations (7) is accompanied by the initial conditions

$$
\tilde{h}(x, 0)=\mathcal{P}\left\{h_{0}(x)\right\}, \quad \tilde{u}(x, 0)=\mathcal{P}\left\{u_{0}(x)\right\},
$$


where $\mathcal{P}$ is the $L^{2}$-projection onto $S$ satisfying $(\mathcal{P} v, \phi)=(v, \phi)$ for all $\phi \in S$. Upon choosing appropriate basis functions for $S,(7)$ is a system of ODEs. For the integration in time of this system, we employ the classical, four-stage, fourth-order explicit Runge-Kutta method.

\section{Solitary waves}

In this section we study how close are the solitary waves of the Euler equations to those of the Serre equations. In other words we verify the consistency of the Serre equations and the ability to approximate well the basic solitary wave dynamics of the Euler equations.

\subsection{Consistency of solitary waves}

The Serre system and the Euler equations both possess solitary waves that decay exponentially to zero at infinity. Although the justification of the Serre equations ensures that its solutions will remain close to Euler solutions, it is not known how close remain an Euler solitary wave to a Serre solitary wave when it is used as initial condition to the Serre system.

While the Serre system admits solitary wave solutions of the form given in (4), there are no known closed-form solitary wave solutions of the Euler system but only Fenton's asymptotic solution [11]. Although this solution is an accurate approximation, modern numerical techniques enable us to compute solitary waves of the Euler equation even more accurately. For this reason we compute Euler solitary waves numerically. The numerical method is a Petviashvili iteration applied to the Babenko equation, [12, 13, 14]. In order to integrate the full Euler equations in time, we employ the method of holomorphic variables. This formulation was first coined by L. Ovsyannikov (1974) [15] and developed later by A. Dyachenko et al. (1996) [16] in deep waters. The extension to the finite depth case was given in [17]. The resulting formulation is discretized in the conformal domain using a Fourier-type pseudo-spectral method. For the time integration we employ an embedded Runge-Kutta scheme of 5(4)th order along with the integrating factor technique to treat the dispersive linear part.

In order to demonstrate the ability of the Serre equations to approximate the Euler equations, we first compare the characteristics of two solitary waves with speeds $c_{s}=1.1$ and $c_{s}=1.2$. The solitary waves are not identical but their differences are small and more pronounced at the higher speed. For example, a $10 \%$ increase in the speed leads to an increase in solitary 
wave amplitude of almost $50 \%$ while the normalized difference between the Euler and Serre solitary waves increased by more than a factor of two. The amplitude of several Euler and Serre solitary waves are presented in Table 1.

\begin{tabular}{lll}
\hline$c_{s}$ & Euler & Serre \\
\hline 1.01 & 0.02012 & 0.0201 \\
1.05 & 0.10308 & 0.1025 \\
1.1 & 0.21276 & 0.2100 \\
1.15 & 0.33007 & 0.3225 \\
1.2 & 0.45715 & 0.4400 \\
1.28 & 0.70512 & 0.6384 \\
\hline
\end{tabular}

Table 1: Amplitudes of the Euler and Serre solitary waves corresponding to different speeds.

Next, we examine how the solitary waves of the Euler system propagate when they are used as initial conditions to the Serre system. Specifically, we use the numerically generated solitary wave solutions of the Euler equations and the exact formula $u=c_{s} \eta /(1+\eta)$ to define the initial conditions $\eta_{0}$ and $u_{0}$ for the Serre equations. Then, we numerically integrate the Serre system. Figures 1 and 2 contain plots of the solutions at $t=150$ obtained using the Euler solitary waves with $c_{s}=1.1$ and $c_{s}=1.2$. These figures demonstrate that the difference between the Euler solitary wave and numerical Serre solution is greater when $c_{s}=1.2$ than when $c_{s}=1.1$. We note that the value $c_{s}=1.2$ is a relatively large value since the largest value we can use to generate an Euler solitary wave is $c_{s}=1.29421$.

To study further the differences between the Euler and Serre solitary waves, we consider three quantities pertinent to the propagation of the solitary waves: the amplitude, shape and phase. First, we define the normalized peak amplitude error as

$$
A E[F] \doteq \frac{\left|F\left(x^{*}(t), t\right)-F(0,0)\right|}{|F(0,0)|},
$$

where $x^{*}(t)$ is the curve along which the computed solution $F(x, t)$ achieves its maximum. Monitoring $A E$ as a function of time, we observe that although the Euler solitary waves do not propagate as traveling waves to the Serre system, their amplitude asymptotically tends towards a constant indicating that they evolve into a solitary wave solution of the Serre equation, see Figures 3 and 4 . 


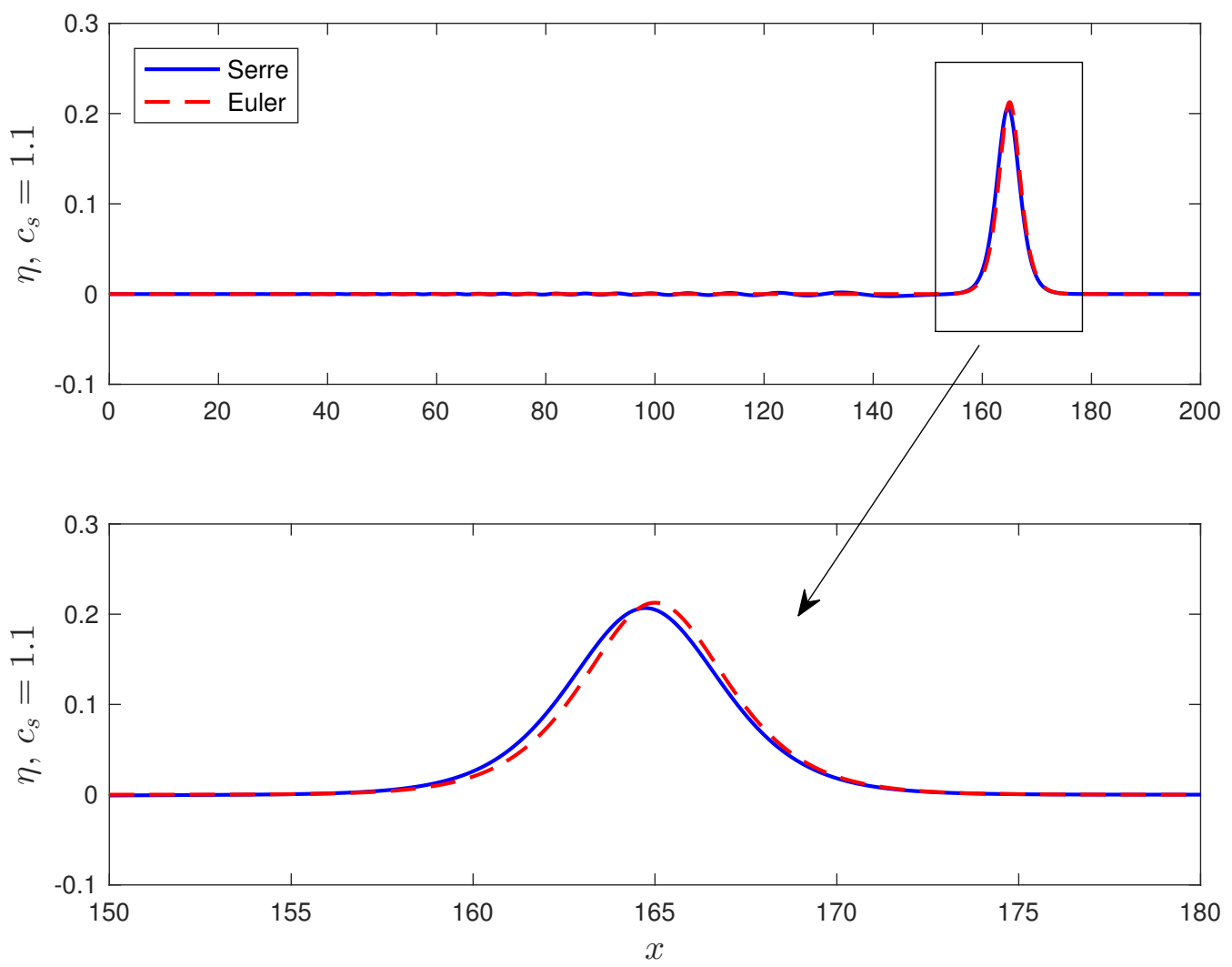

Figure 1: The evolution of an Euler solitary wave with $c_{s}=1.1$ when used as initial condition in the Serre system. 


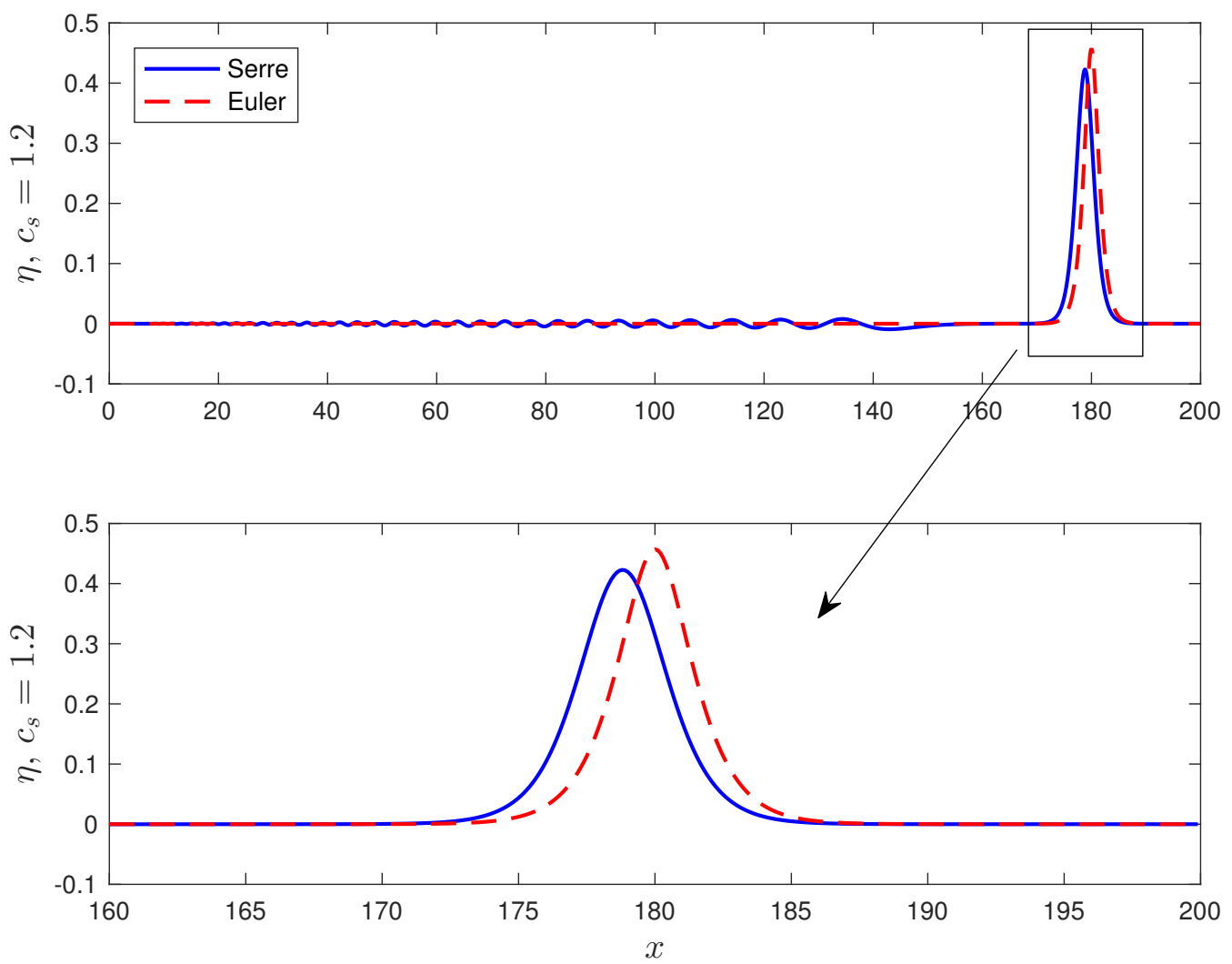

Figure 2: The evolution of an Euler solitary wave with $c_{s}=1.2$ when used as initial condition in the Serre system. 

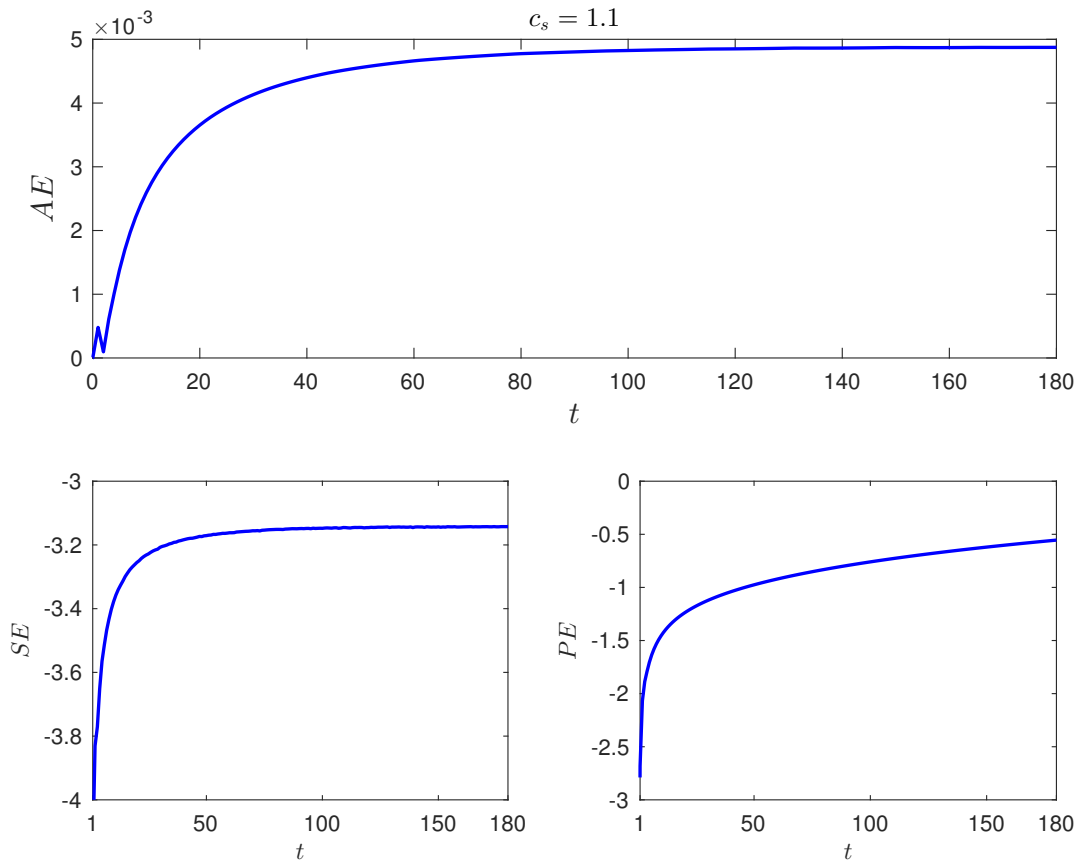

Figure 3: The amplitude, shape and phase error of the Euler's solitary wave of $c_{s}=1.1$ propagating with Serre equations. See also Figure 1. 

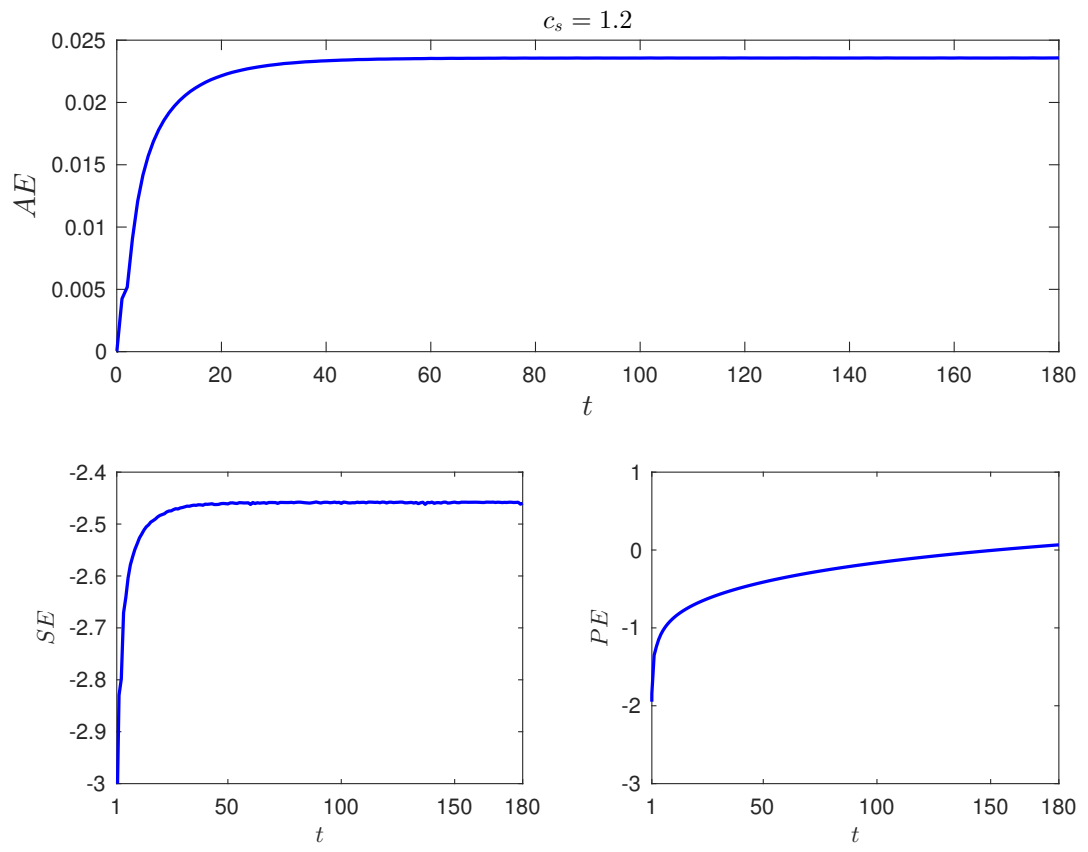

Figure 4: The amplitude, shape and phase errors of the Euler's solitary wave of $c_{s}=1.1$ propagating with Serre equations. See also Figure 2. 
We define the normalized shape error as

$$
S E[F] \doteq \log _{10}\left(\min _{\tau} \zeta(\tau)\right), \quad \zeta(\tau) \doteq \frac{\left\|F\left(x, t^{n}\right)-F_{\text {exact }}(x, \tau)\right\|}{\left\|F_{\text {exact }}(x, 0)\right\|} .
$$

The minimum in (11) is attained at some critical $\tau=\tau^{*}\left(t^{n}\right)$. This, in turn, is used to define the phase error as

$$
P E[F] \doteq \log _{10}\left(\left|\tau^{*}-t^{n}\right|\right)
$$

In order to find $\tau^{*}$, we use Newton's method to solve the equation $\zeta^{\prime \prime}(\tau)=0$. The initial guess for Newton's method is chosen as $\tau^{0}=t^{n}-\Delta t$. Figures 3 and 4 contain plots of the shape and phase errors. We observe that the shape error is of $\mathcal{O}\left(10^{-3}\right)$ when $c_{s}=1.1$ and of $\mathcal{O}\left(10^{-2}\right)$ when $c_{s}=1.2$. The phase error increases since the solitary waves propagate with different speeds. It is remarkable that the phase speeds of the new solitary waves of the Serre system are almost the same as the phase speeds of the Euler's solitary waves. For example, the speeds are $c_{s} \approx 1.09$ and $c_{s} \approx 1.19$. Similar comparisons have been performed for other model equations such as the classical Boussinesq system and the results are comparable [18, 19].

\subsection{Head-on collision of solitary waves}

The collision of two solitary waves of the Serre system has previously been studied theoretically and numerically in $[9,10,17,20,21,22,23,24]$. While the phenomena related to these interactions have been understood quite well we summarize here the dynamics of the head-on collision of solitary waves and we focus on the related dynamics compared to experimental data and to numerical simulations of the full Euler equations. The interaction of solitary waves for the Serre equations is general more inelastic than in weakly nonlinear models such as the classical Boussinesq system [18]. Highly nonlinear interactions result in the generation of large amplitude dispersive tails.

In order to study the physical relevance of the head-on collision of two Serre solitary waves, we compare the Serre numerical solution with the experimental data of [23]. In this experiment, the Serre system is written in dimensional and unscaled form with an initial condition that includes two counter-propagating solitary waves in the interval $[-5,5]$. The speeds of these solitary waves are $c_{s, 1}=0.7721 \mathrm{~m} / \mathrm{s}$ and $c_{s, 2}=0.7796 \mathrm{~m} / \mathrm{s}$. Their amplitudes are $A_{1}=0.0108 \mathrm{~m}$ and $A_{2}=0.0120 \mathrm{~m}$ respectively. (In this 

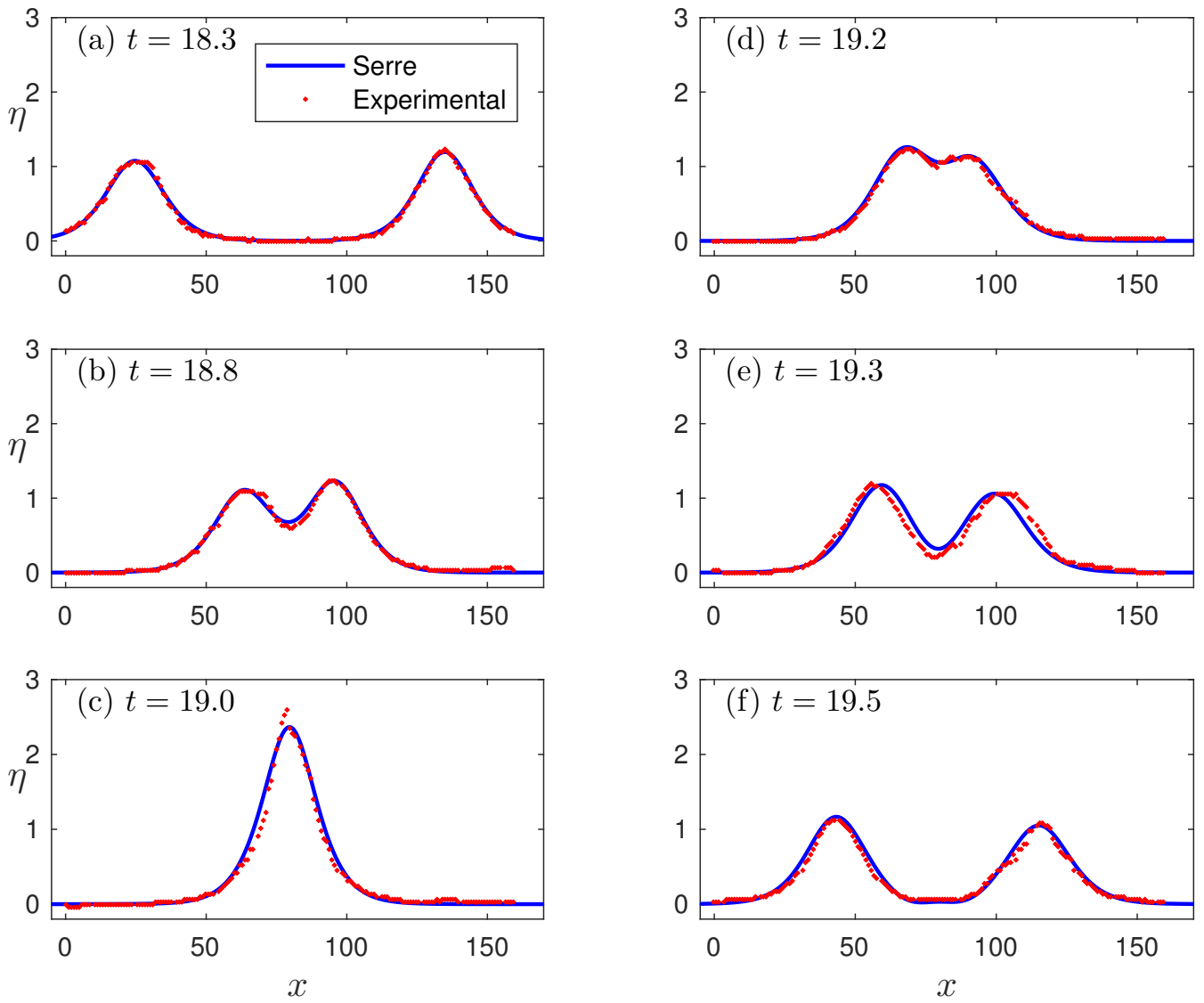

Figure 5: Comparison of the head-on collision of two solitary waves of the Serre system with experimental data. 

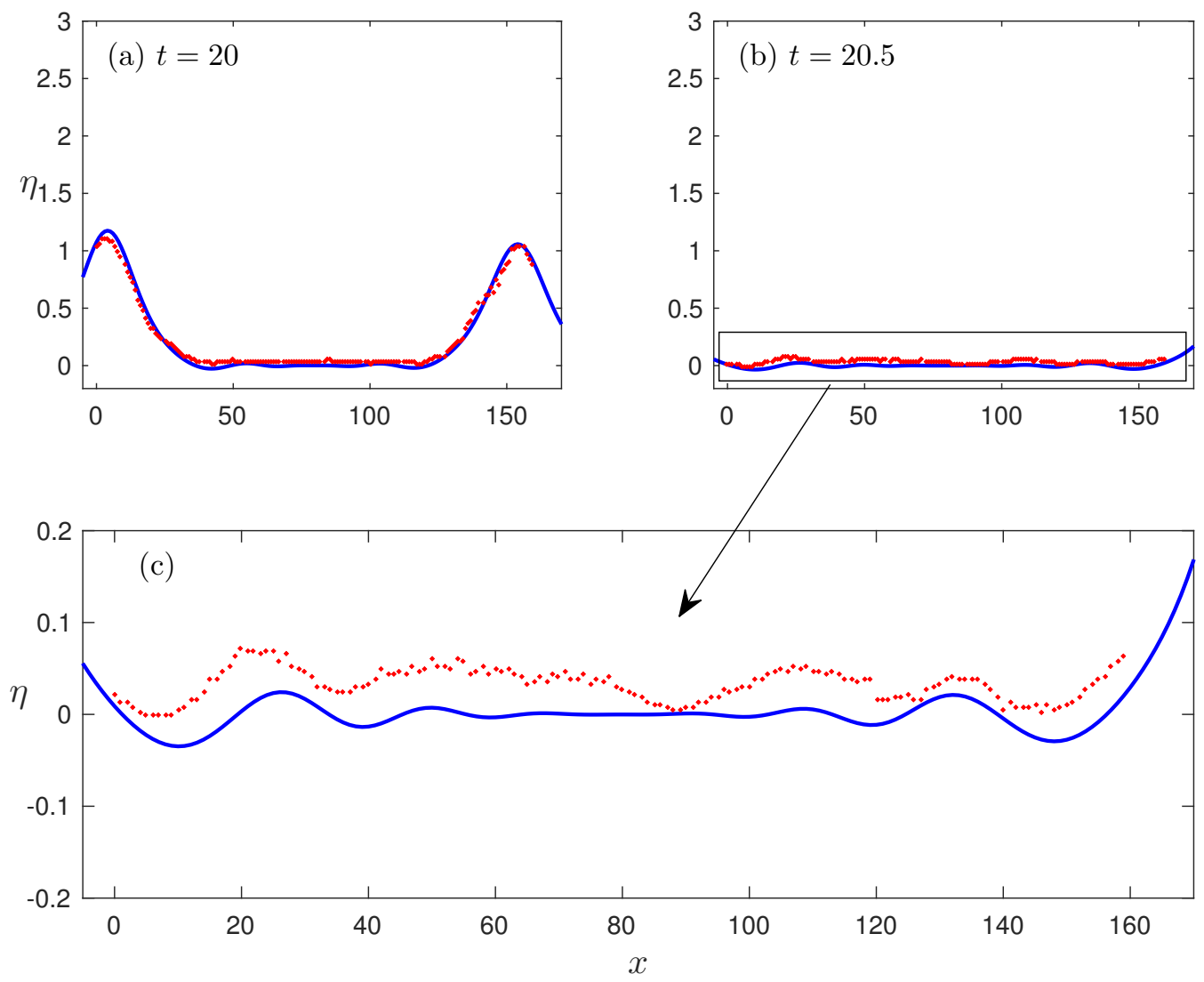

Figure 6: (Cont'd) Comparison of the head-on collision of two solitary waves of the Serre system with experimental data.

experiment the depth $d=0.05 \mathrm{~m}$.). At $t=18.3 \mathrm{~s}$ these solitary waves achieved their maximum values at $x_{1}=0.247 \mathrm{~m}$ and $x_{2}=1.348 \mathrm{~m}$ respectively. Figures 5 and 6 include comparisons between the numerical solution and experimental data. The agreement between the numerical results and the experimental data is impressive. The agreement in the generated dispersive tails in Figure 6 is even more impressive. Such agreement cannot be found in the case of head-on collisions of solitary waves of Boussinesq type models, [25], indicating that the high-order nonlinear terms are important in studying even these small-amplitude solutions. Finally, we mention that the maximum amplitude of the solution observed in Figure 5(c) during the collision is smaller than the real amplitude, possibly, because of a splash phenomenon that cannot be described by any model (see also [25]). 
We now compare a head-on collision of two unequal solitary waves via numerical solutions of the Serre and Euler equations. For both models, we consider a right-traveling solitary wave with $c_{s}=1.1$ and a left-traveling solitary wave with $c_{s}=1.2$. These solitary waves are initially translated so that the maximum peak amplitudes are achieved at $x=-100$ and $x=100$ respectively. Results from the numerical simulations are included in Figure 7. Both models show similar behavior, however the maximum amplitude observed during the collision using the Euler equations is larger than in the Serre system. Also the interaction in the Euler equations lasts longer and therefore a larger phase shift is observed. The leading waves of the dispersive tails are almost identical in the two models, but the amplitude of the tails in the Euler system decay to zero more slowly than the amplitude of the tails in the Serre system. These numerical simulations verify the ability of the Serre system to accurately model head-on collisions of solitary waves. They also show that the Serre system is consistent with the Euler equations during and after the head-on collision with almost identical solutions.

\section{Stability of traveling waves}

The previous experiment of the head-on collision of two solitary waves indicates that the solitary waves are robust. In this section, we present the behavior of a solitary wave under small perturbations. We explore the effects of modifying some of the high-order terms of the Serre system. We show that modifying one such term one can produce regularized shock waves, as opposed

to classical dispersive shock waves. Finally, we examine the stability of the cnoidal wave solutions.

\subsection{Stability of solitary waves}

We consider perturbations of the amplitude, perturbations of the wavelength, and random-noise perturbations of the shape. As we show below, all of the solitary waves we tested were stable to all of the perturbations we considered.

We chose a solitary wave with speed $c_{s}=1.4$ and amplitude $A=0.96$ for all numerical simulations in this section. We perturb the amplitude by multiplying the pulse by a parameter $p$ such that

$$
h_{p}(x, 0)=1+p \cdot a_{1} \operatorname{sech}^{2}\left(K_{s} x\right),
$$



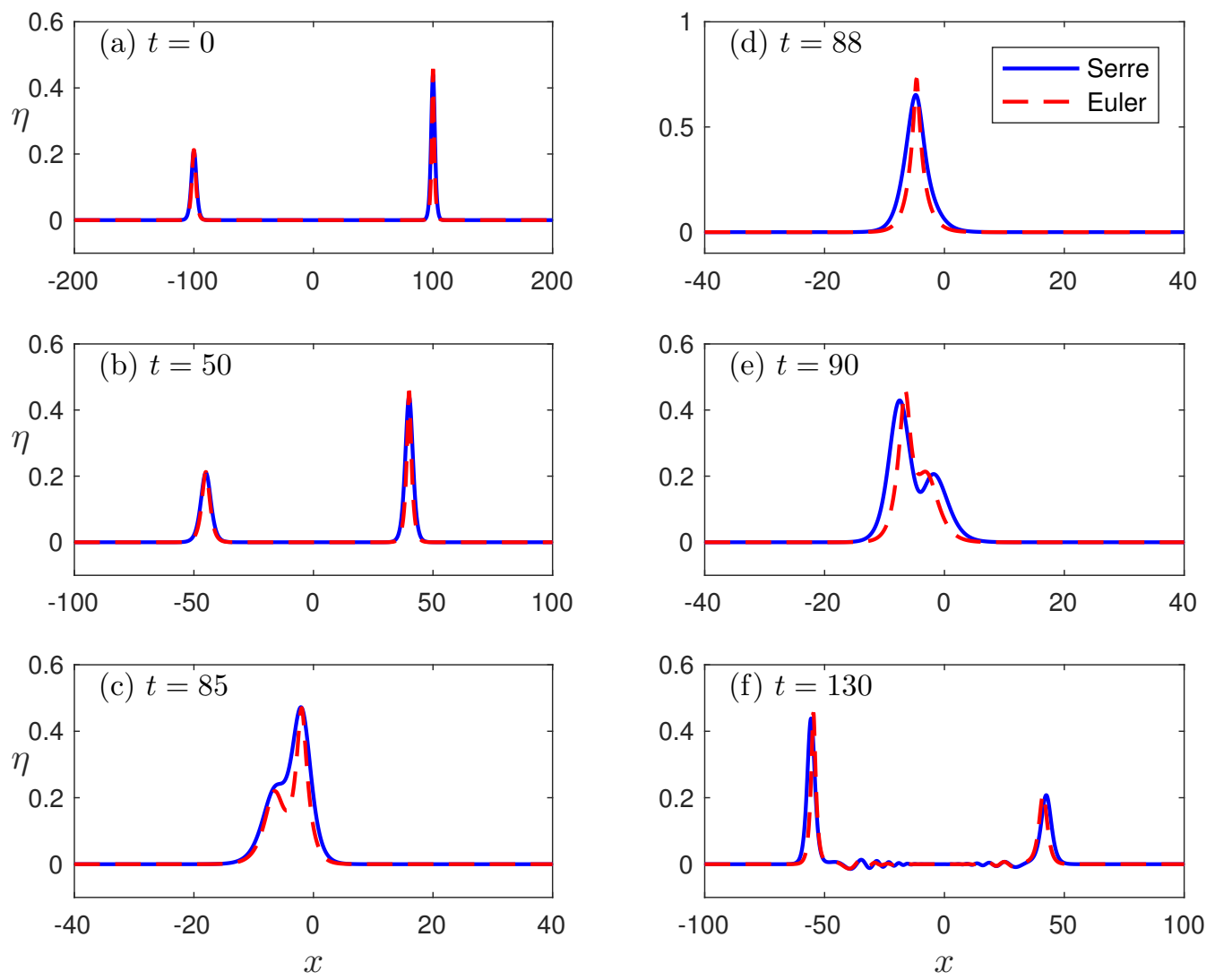

Figure 7: Comparison between the head-on collisions of two solitary waves for the Serre and Euler systems. 
while keeping the velocity component of the solution unperturbed as in (4). When $p=1.1$ the initial condition sheds a small-amplitude dispersive tail and results in a new solitary wave with amplitude $A=1.02050$. Figure 8 presents the initial condition and the resulting solution at $t=130$. Similar observations resulted in all cases we tested.

We consider perturbations of the wavelength $K_{s}$ by taking the initial condition for $h$ to be

$$
h_{p}(x, 0)=1+a_{1} \operatorname{sech}^{2}\left(p \cdot K_{s} x\right) .
$$

The results in this case were very similar to the results we obtained when we perturbed the amplitude of the solitary waves and so we don't show the results here. Table 2 shows the amplitudes of the solitary waves that result from various amplitude and wavelength perturbations.

\begin{tabular}{lll}
\hline$p$ & Amplitude perturbation & Wavelength perturbation \\
\hline 0.8 & 0.83860 & 1.02710 \\
0.9 & 0.89936 & 0.99225 \\
1.1 & 1.02050 & 0.93017 \\
1.2 & 1.08087 & 0.90260 \\
\hline
\end{tabular}

Table 2: Amplitudes of the uniformly perturbed solitary waves.

Similar results were obtained when non-uniform perturbations were used. In order to consider non-uniform perturbations, we used pseudo-random noise distributed uniformly in $[0,1]$. Denoting the noise function by $N(x)$, the perturbed solitary wave is given by

$$
h_{p}(x, t)=1+(1-p N(x)) \cdot a_{1} \operatorname{sech}^{2}\left(K_{s} x-c_{s} t\right),
$$

where the parameter $p$ determines the magnitude of the noise. Figure 9 shows the perturbed solitary wave with $p=0.2$. This type of perturbation is not only non-uniform, but is also non-smooth. Nevertheless this initial condition is the $L^{2}$-projection of the actual solution which ensures the required by the FEM smoothness. Figure 10 shows the evolution of this perturbed solitary wave. The solution consists of a new solitary wave and a small-amplitude dispersive tail. It does not differ qualitatively from the solution shown in Figure 8 . The values of the amplitudes of the emerging solitary waves for various values of $p$ are presented in Table 3. These results suggest that the solitary waves of the Serre system are orbitally stable with respect to this class of perturbations. 


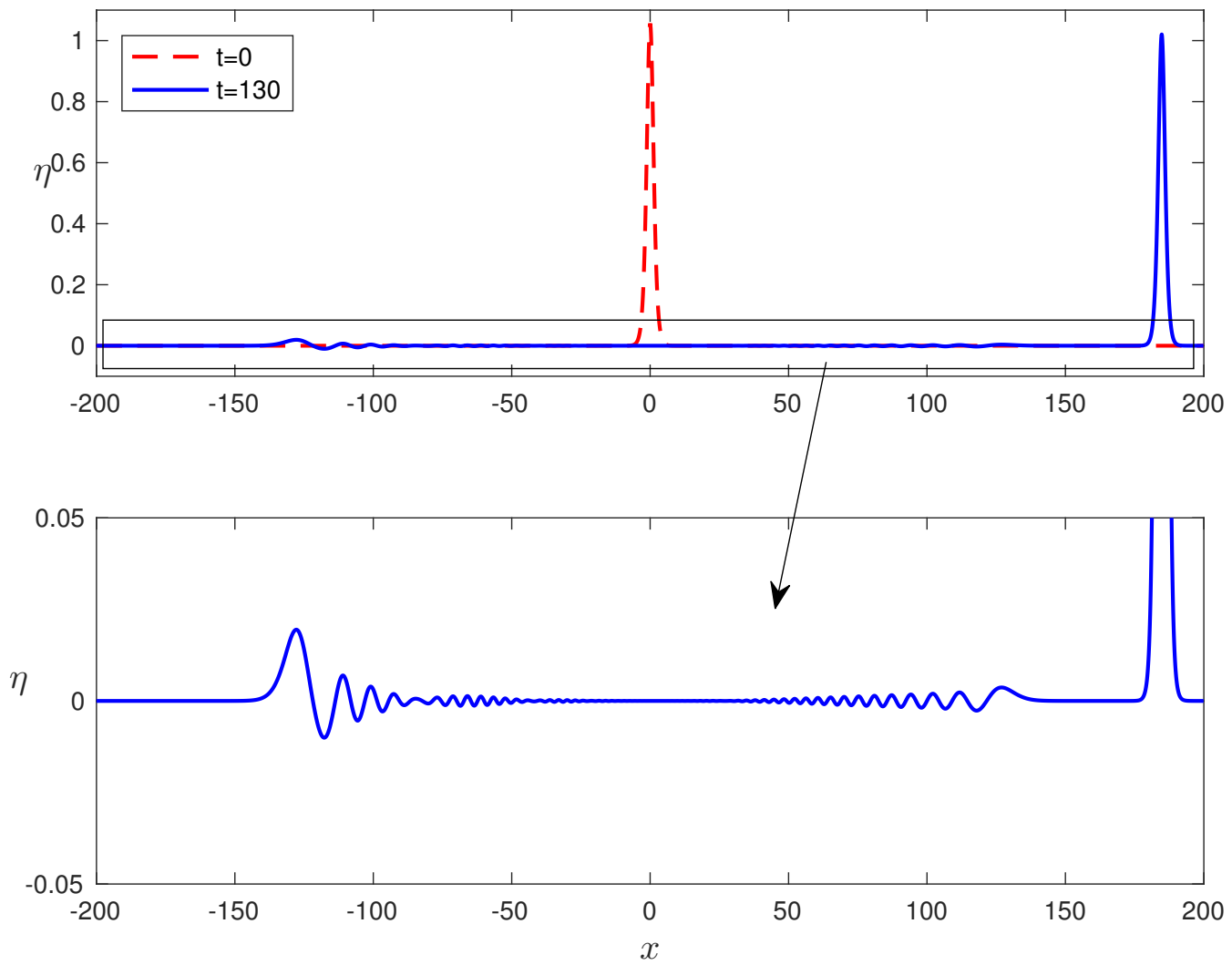

Figure 8: The perturbed solution generated by the perturbation of the amplitude of the solitary wave with $c_{s}=1.4, A=0.96$, and perturbation parameter $p=1.1$.

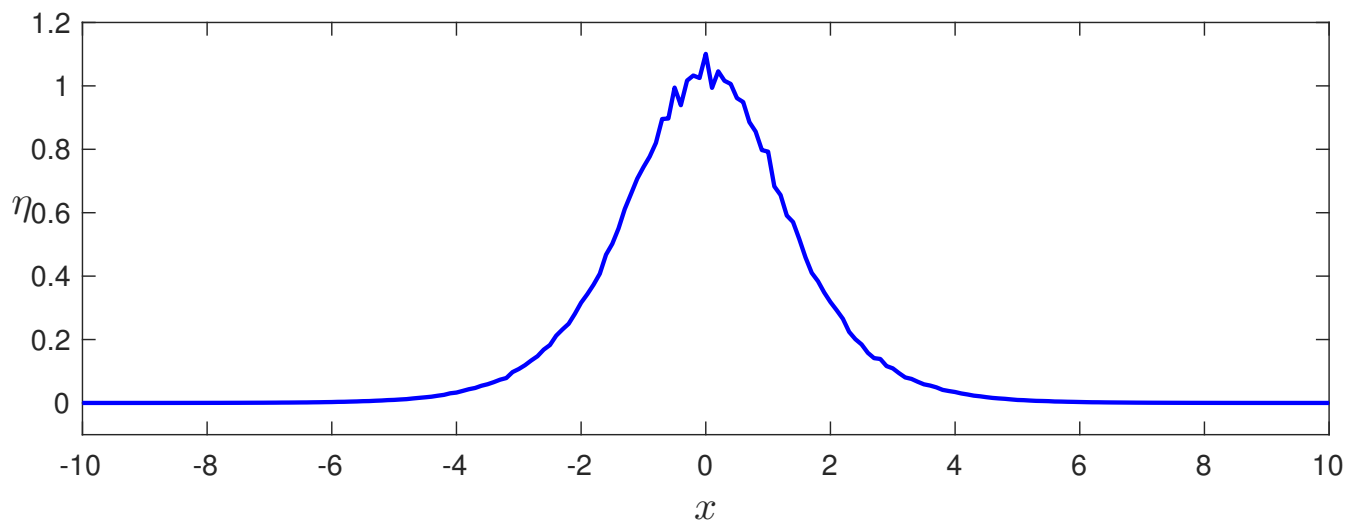

Figure 9: The solitary wave perturbed by pseudo-random noise with $p=0.2$. 


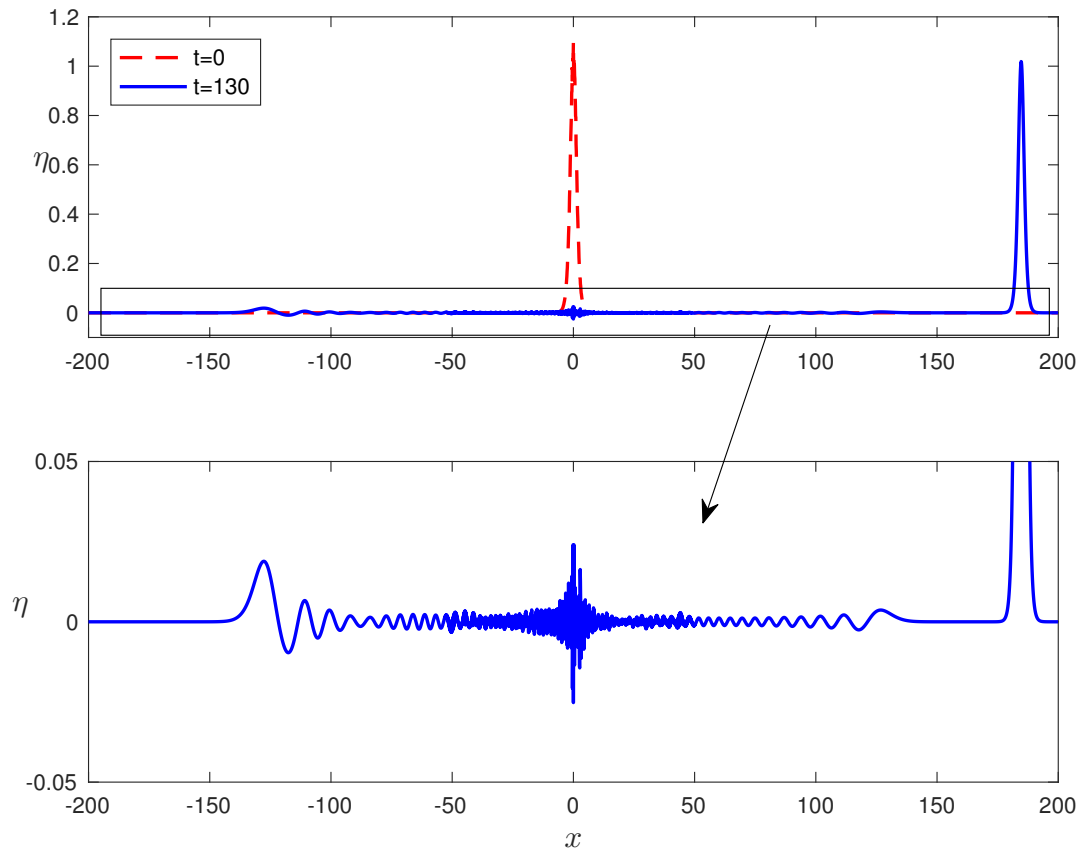

Figure 10: Evolution of the initial condition shown in Figure 9 with $p=0.2$.

\subsection{Persistence of the solitary waves}

One other aspect related to the stability of the solitary waves is their ability to persist when some of the high-order terms in the PDE are perturbed. In this section, we examine if a Serre solitary wave retains its shape when some of the terms of the Serre system are perturbed. Introducing the parameters $\alpha, \beta$, and $\gamma$, we rewrite the Serre equations in the following form

$$
\begin{aligned}
& \eta_{t}+u_{x}+(\eta u)_{x}=0, \\
& u_{t}+\eta_{x}+u u_{x}-\frac{1}{3 h}\left[h^{3}\left(\alpha u_{x t}+\beta u u_{x x}-\gamma\left(u_{x}\right)^{2}\right)\right]_{x}=0 .
\end{aligned}
$$

The unperturbed Serre equations correspond to $\alpha=\beta=\gamma=1$. We first study the persistence of the solitary waves when the system is perturbed by perturbing the parameters $\alpha, \beta, \gamma$ and considering a solitary wave of the unperturbed system as an initial condition. In this section we use the solitary wave (4) with $c_{s}=1.4$ as an initial condition. If $\alpha=0.9$, or if $\beta=0.9$, or if $\gamma=0.9$ the solitary wave evolves in a manner similar to the amplitude perturbations in Section 4.1. The new solitary waves are very similar to the unperturbed solitary wave. This further indicates that the solitary waves of the Serre system are stable. 


\begin{tabular}{ll}
\hline$p$ & Amplitude \\
\hline-0.2 & 0.90110 \\
-0.1 & 0.93055 \\
0.1 & 0.98942 \\
0.2 & 1.01883 \\
0.5 & 1.10694 \\
\hline
\end{tabular}

Table 3: Amplitudes of the randomly perturbed solitary waves.

More interesting phenomena is observed when the solitary waves are used as initial conditions to systems with small values of the parameters $\alpha, \beta$ and $\gamma$. When all the three parameters are very small, the solutions tend to break into dispersive shock waves or other forms of undular bores. In the first numerical simulation, we consider $\alpha=\beta=\gamma=0.01$. This is similar to the case of the small dispersion limit where the weakly nonlinear terms are dominant. Figure 11 demonstrates that the solution becomes a dispersive shock. This phenomenon has been previously observed in dispersive systems, cf. $[25,26,27]$. Unexpectedly, taking the parameters $\beta$ and $\gamma$ to be very small, i.e. $\beta=\gamma=0.001$ and keeping the parameter $\alpha=1$, the solitary wave persists and evolves into a new solitary wave which is similar to the unperturbed solitary wave qualitatively similar to those presinted in Section 4.1. This persistence is remarkable because the solitary wave remains almost the same even if two of the most important terms have been almost eliminated. If $\alpha=\beta=1$ and $\gamma=0.001$ or if $\alpha=\gamma=1$ and $\beta=0.001$ the behavior is similar.

The behavior changes dramatically if large perturbations $\alpha$ are considered. The results from the simulation with $\alpha=0.001$ and $\beta=\gamma=1$ is shown in Figure 12. In this case, the initial condition breaks into different waves but instead of forming a dispersive shock wave, it forms a new kind of regularized shock wave. This suggests a new breaking mechanism by the elimination of the $u_{x t}$ term. Similar dissipative behavior has been observed in nonlinear $\mathrm{KdV}$-type equations where some high-order nonlinear terms introduce dissipation to the system [28].

\subsection{Stability of Cnoidal waves}

We follow the work of Carter \& Cienfuegos [29] in order to study the linear stability of the solutions given in (5). We enter a coordinate frame moving with the speed of the solutions by defining $\chi=x-c_{c} t$ and $\tau=t$. 

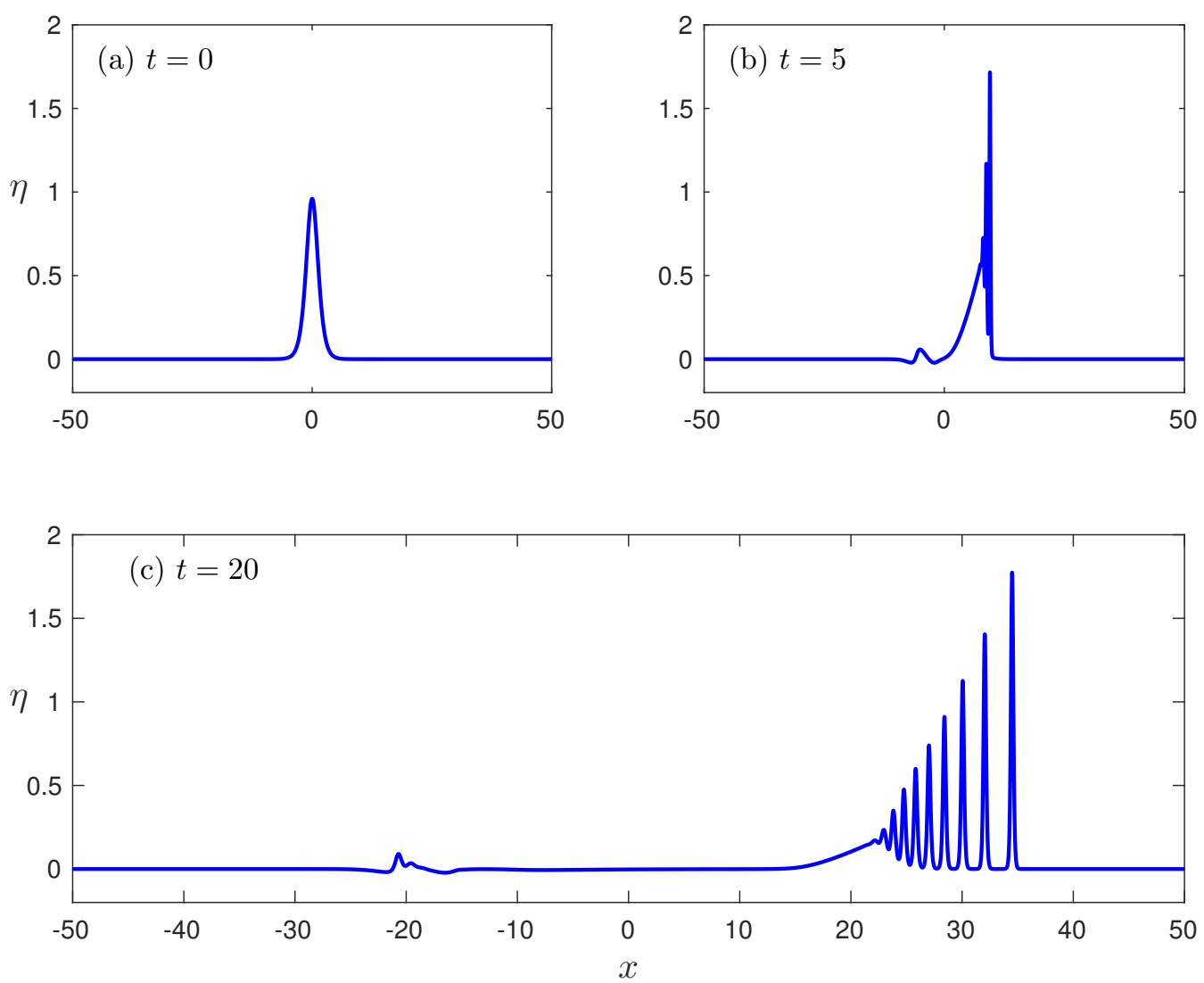

Figure 11: The evolution of a solitary wave of a perturbed system ( $\alpha=\beta=\gamma=$ $0.01)$. 

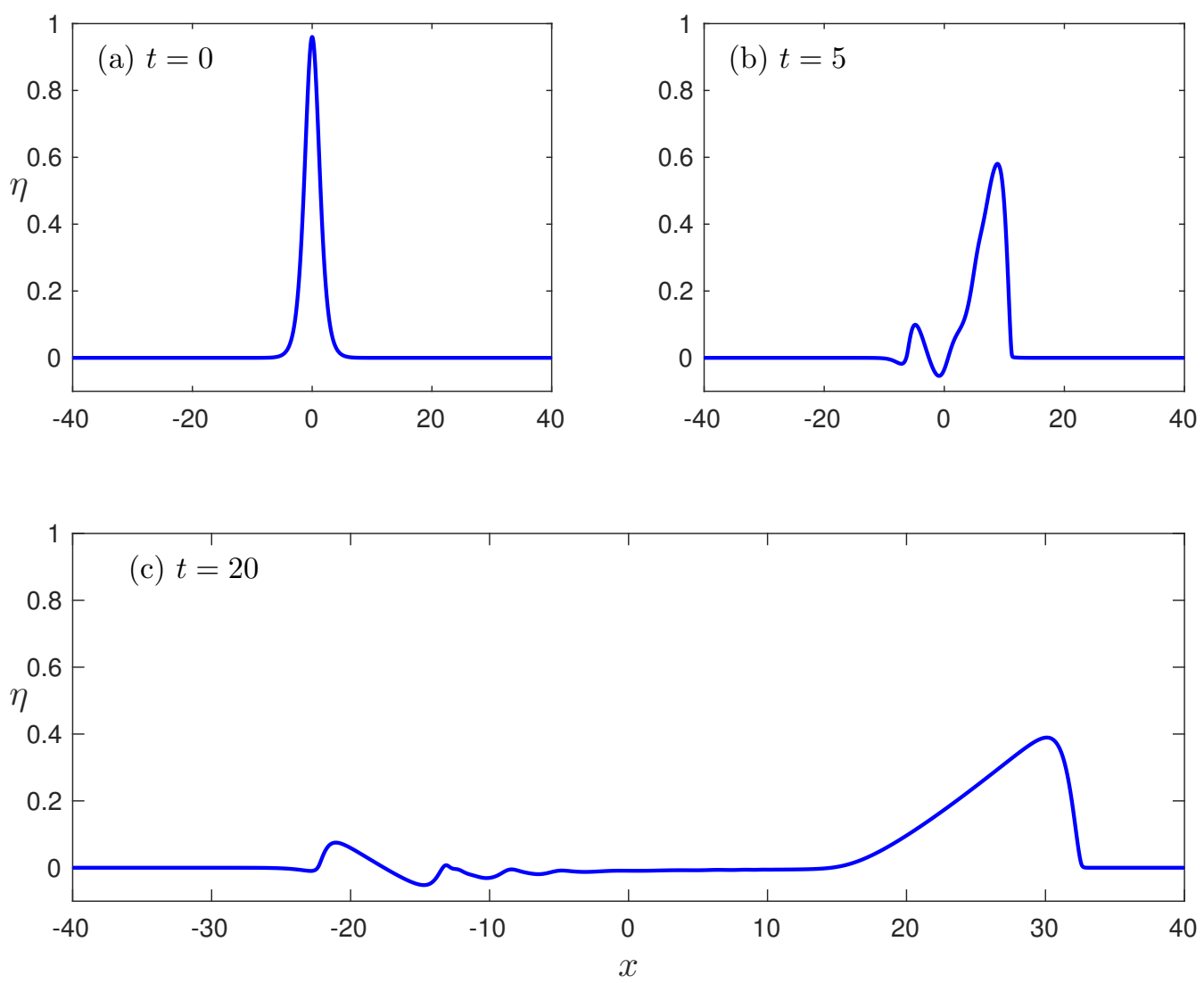

Figure 12: The evolution of a solitary wave of a perturbed system and the generation of a regularized shock $(\alpha=0.001, \beta=\gamma=1)$. 
In this moving frame, the Serre equations are given by

$$
\begin{aligned}
& h_{\tau}-c_{c} h_{\chi}+(h u)_{\chi}=0 \\
& u_{\tau}-c_{c} u_{\chi}+u u_{\chi}+h_{\chi}-\frac{1}{3 h}\left(h^{3}\left(u_{\chi \tau}-c_{c} u_{\chi \chi}+u u_{\chi \chi}-\left(u_{\chi}\right)^{2}\right)\right)_{\chi}=0
\end{aligned}
$$

and the solution given in (5) simplifies to the following time-independent solution

$$
\begin{aligned}
& h=h_{0}(\chi)=a_{0}+a_{1} \operatorname{dn}^{2}\left(K_{c} \chi, k\right), \\
& u=u_{0}(\chi)=c_{c}\left(1-\frac{h_{0}}{h(\chi)}\right) .
\end{aligned}
$$

We consider perturbed solutions of the form

$$
\begin{aligned}
& h_{\text {pert }}(\chi, \tau)=h_{0}(\chi)+\mu h_{1}(\chi, \tau)+\mathcal{O}\left(\mu^{2}\right), \\
& u_{\text {pert }}(\chi, \tau)=u_{0}(\chi)+\mu u_{1}(\chi, \tau)+\mathcal{O}\left(\mu^{2}\right),
\end{aligned}
$$

where $h_{1}$ and $u_{1}$ are real-valued functions and $\mu$ is a small real parameter. Substituting (19) into (17) and linearizing leads to a pair of coupled, linear partial differential equations that are constant coefficient in $\tau$. Without loss of generality, assume

$$
\begin{aligned}
& h_{1}(\chi, \tau)=H(\chi) \mathrm{e}^{\Omega \tau}+\text { c.c. } \\
& u_{1}(\chi, \tau)=U(\chi) \mathrm{e}^{\Omega \tau}+\text { c.c. }
\end{aligned}
$$

where $H(\chi)$ and $U(\chi)$ are complex-valued functions, $\Omega$ is a complex constant, and c.c. denotes complex conjugate. If $\Omega$ has a positive real part, i.e. if $\operatorname{Re}(\Omega)>0$, then the perturbations $h_{1}$ and $u_{1}$ grow exponentially in $\tau$ and the solution is said to be unstable.

Substituting (20) into the linearized PDEs gives

$$
\mathcal{L}\left(\begin{array}{c}
H \\
U
\end{array}\right)=\Omega \mathcal{M}\left(\begin{array}{c}
H \\
U
\end{array}\right)
$$

where $\mathcal{L}$ and $\mathcal{M}$ are the linear differential operators defined by

$$
\begin{aligned}
& \mathcal{L}=\left(\begin{array}{cc}
-u_{0}^{\prime}+\left(c_{c}-u_{0}\right) \partial_{\chi} & -\eta_{0}^{\prime}-\eta_{0} \partial_{\chi} \\
\mathcal{L}_{21} & \mathcal{L}_{22}
\end{array}\right), \\
& \mathcal{M}=\left(\begin{array}{ccc}
1 & 0 \\
0 & 1-\eta_{0} \eta_{0}^{\prime} \partial_{\chi}-\frac{1}{3} \eta_{0}^{2} \partial_{\chi \chi}
\end{array}\right)
\end{aligned}
$$


where prime represents derivative with respect to $\chi$ and

$$
\begin{aligned}
\mathcal{L}_{21}= & -\eta_{0}^{\prime}\left(u_{0}^{\prime}\right)^{2}-c_{c} \eta_{0}^{\prime} u_{0}^{\prime \prime}-\frac{2}{3} c_{c} \eta_{0} u_{0}^{\prime \prime \prime}+\eta_{0}^{\prime} u_{0} u_{0}^{\prime \prime}-\frac{2}{3} \eta_{0} u_{0}^{\prime} u_{0}^{\prime \prime}+ \\
& \frac{2}{3} \eta_{0} u_{0} u_{0}^{\prime \prime \prime}+\left(\eta_{0} u_{0} u_{0}^{\prime \prime}-g-\eta_{0}\left(u_{0}^{\prime}\right)^{2}-c_{c} \eta_{0} u_{0}^{\prime \prime}\right) \partial_{\chi}, \\
\mathcal{L}_{22}= & -u_{0}^{\prime}+\eta_{0} \eta_{0}^{\prime} u_{0}^{\prime \prime}+\frac{1}{3} \eta_{0}^{2} u_{0}^{\prime \prime \prime}+\left(c_{c}-u_{0}-2 \eta_{0} \eta_{0}^{\prime} u_{0}^{\prime}-\frac{1}{3} \eta_{0}^{2} u_{0}^{\prime \prime}\right) \partial_{\chi}+ \\
& \left(\eta_{0} \eta_{0}^{\prime} u_{0}-c_{c} \eta_{0} \eta_{0}^{\prime}-\frac{1}{3} \eta_{0}^{2} u_{0}^{\prime}\right) \partial_{\chi \chi}+\left(\frac{1}{3} \eta_{0}^{2} u_{0}-\frac{1}{3} c_{c} \eta_{0}^{2}\right) \partial_{\chi \chi \chi} .
\end{aligned}
$$

The Fourier-Floquet-Hill method described in Deconinck \& Kutz [30] is then used to solve the differential eigenvalue problem given in (21). This method establishes that all bounded solutions of (21) have the form

$$
\left(\begin{array}{c}
H \\
U
\end{array}\right)=\mathrm{e}^{\mathrm{i} \rho \chi}\left(\begin{array}{c}
H^{P} \\
U^{P}
\end{array}\right),
$$

where $H^{P}$ and $U^{P}$ are periodic in $\chi$ with period $2 K / K_{c}$ and $\rho \in\left[-\pi K_{c} /(4 K), \pi K_{c} /(4 K)\right]$.

Using this method, Carter \& Cienfuegos established that solutions of the form given in (18) with sufficiently small amplitude and steepness are spectrally stable and solutions with sufficiently large amplitude or steepness are spectrally unstable. For example, the solution with $a_{0}=0.3, a_{1}=0.2$ and $k=0.75$ is unstable with respect to the perturbation shown in Figure 13. The period of this perturbation is twelve times the period of the exact solution $(\rho=1 / 12)$. The theory establishes that the magnitude of this perturbation will grow like $\mathrm{e}^{0.00569 t}$. We corroborated this result by using the following perturbation-seeded solution as the initial condition in our Serre solver

$$
\begin{aligned}
& h_{\text {pert }}(x, 0)=h(x, 0)+10^{-7} h_{1}(x), \\
& u_{\text {pert }}(x, 0)=u(x, 0)+10^{-7} u_{1}(x) .
\end{aligned}
$$

Here $(h(x, 0), u(x, 0))$ is the solution given in equations (18) with $a_{0}=0.3$, $a_{1}=0.2$ and $k=0.75$ and $\left(h_{1}(x), u_{1}(x)\right)$ is the perturbation shown in Figure 13. Figure 14 contains a plot of the magnitude of the first Fourier mode of the solution versus $t$. This mode initially (up to $t=1500$ ) grows exponentially with a rate of 0.00577 , very close to the rate predicted by the linear theory. However after more time, the solution returns to a state close to the initial one. The first portion of this recurrence phenomenon is depicted in Figure 15 . Note that the solution at $t=2870$ has nearly returned to its initial state. Similar behavior has been observed to other shallow water models by Ruban [31] and it is referred to as the Fermi-Pasta-Ulam recurrence. 

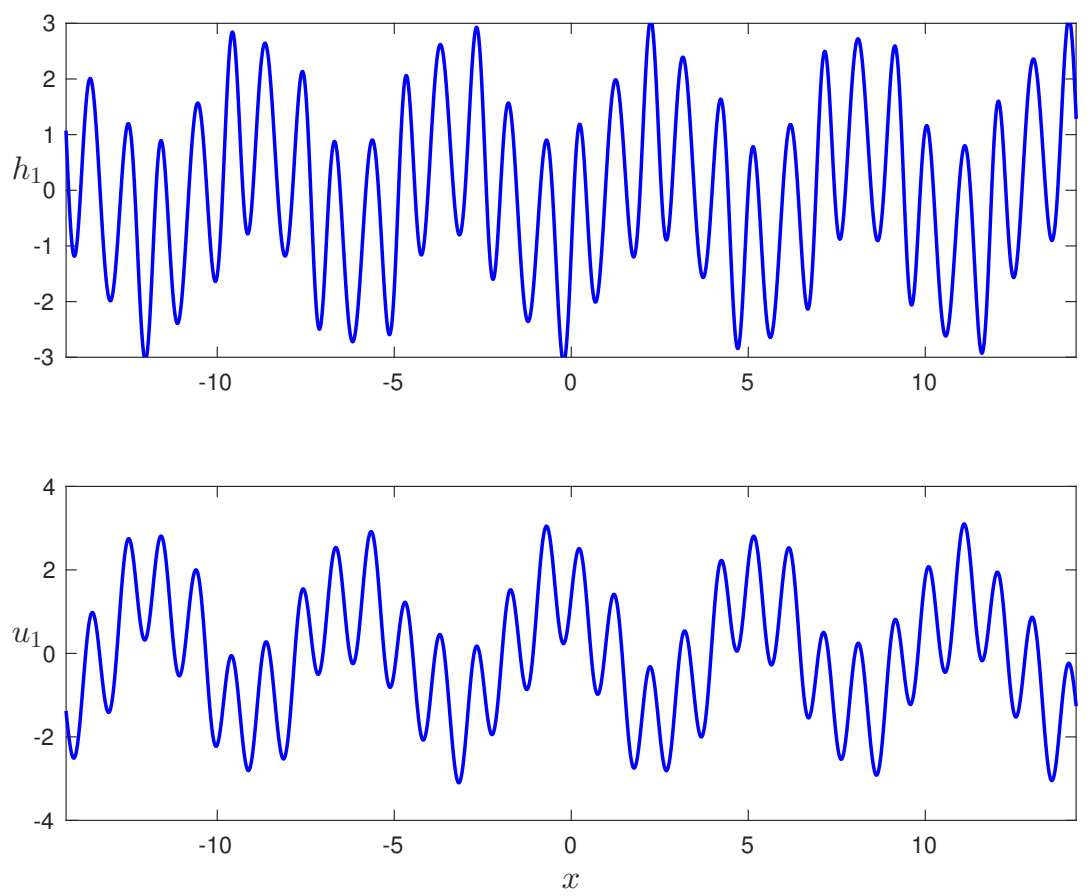

Figure 13: Unstable perturbations corresponding to the solution given in (5) with $a_{0}=0.3, a_{1}=0.2$ and $k=0.75$.

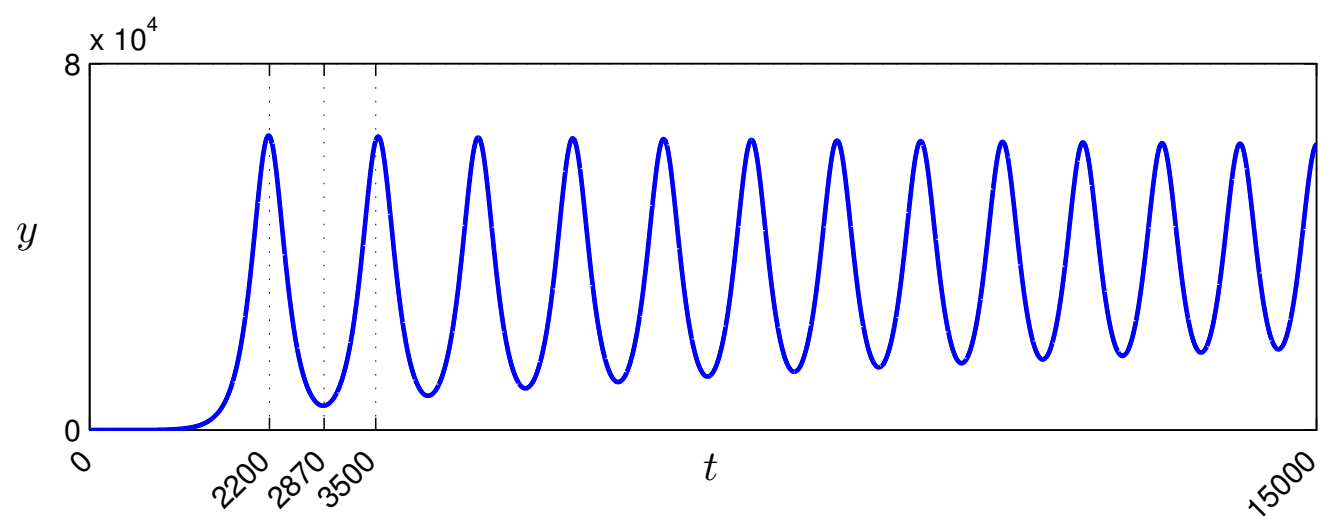

Figure 14: The magnitude of the first Fourier mode of the perturbation versus time. 

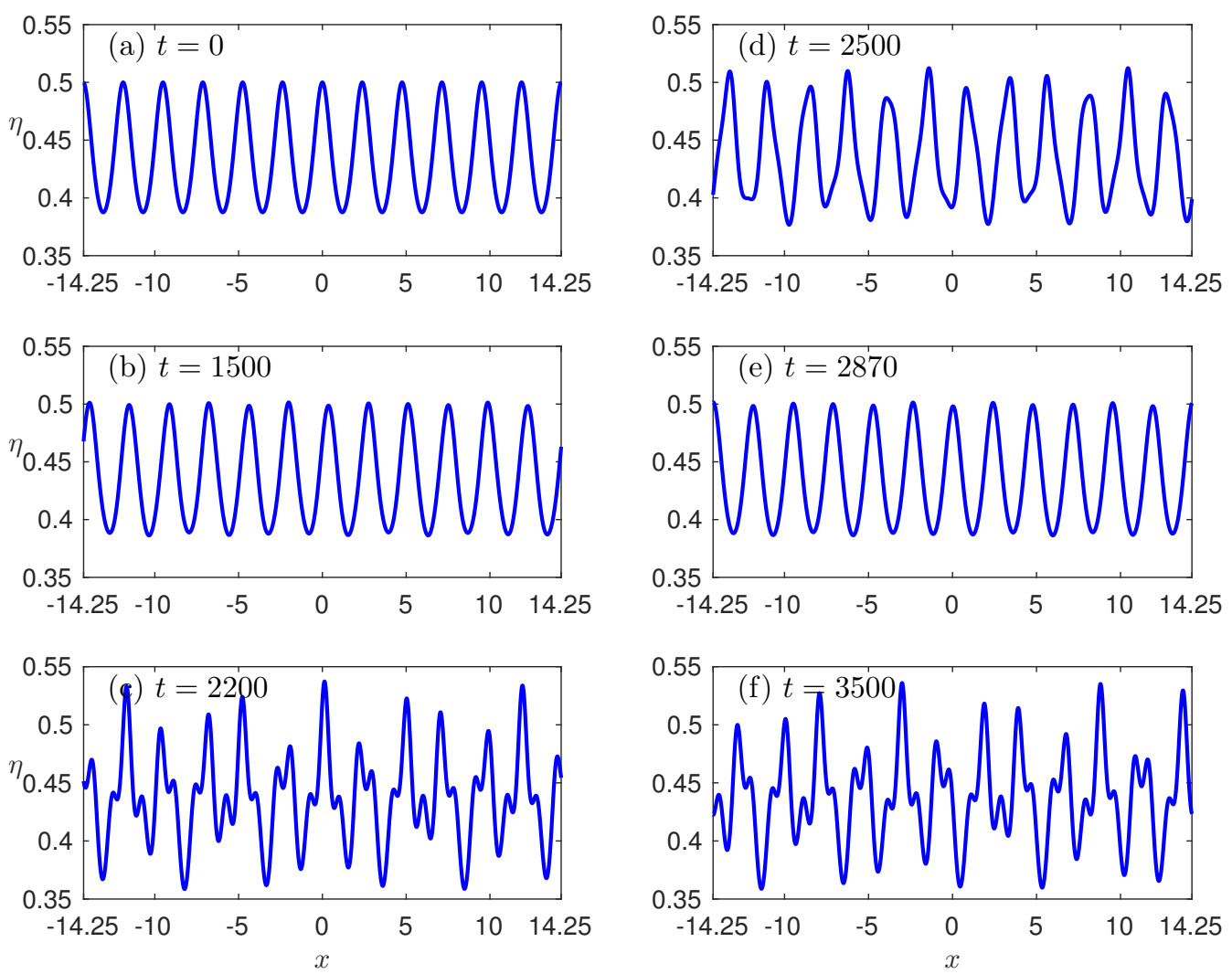

Figure 15: The periodic instability of the perturbed cnoidal wave. 


\section{Dispersive shock waves}

A simple DSW traveling to the right can be generated using the Riemann initial data, cf. [26],

$$
h(x, 0)=\left\{\begin{array}{ll}
h^{-}, & \text {for } x<0 \\
h^{+}, & \text {for } x>0
\end{array}, \quad u(x, 0)=\left\{\begin{array}{ll}
u^{-}, & \text {for } x<0 \\
u^{+}, & \text {for } x>0
\end{array},\right.\right.
$$

with the compatibility condition (Riemann invariant)

$$
\frac{u^{-}}{2}-\sqrt{h^{-}}=\frac{u^{+}}{2}-\sqrt{h^{+}}
$$

DSWs can also be generated during the dam-break problem simulation. In this case, the initial data for $h(x, 0)$ are the same as in $(27)$, but there is no flow at $t=0$, i.e. , $u(x, 0)=0$. As shown in [26], this generates two counterpropagating DSWs, one on each side of the "dam", and two rarefaction waves that travel toward the center. We consider the initial condition for $h$ to be a smooth step function that decays to zero as $|x| \rightarrow \infty$. Specifically, we choose

$$
\eta(x, 0)=\frac{1}{2} \eta_{0}\left[1+\tanh \left(\frac{x_{0}-|x|}{2}\right)\right]
$$

where $\eta_{0}=0.1, x_{0}=350$, and $u(x, 0)=0$. A plot of this initial condition is included in Figure 16. Both the Euler and Serre equations generate two counter propagating DSWs and two rarefaction waves. Figure 16 demonstrates that the amplitude of the leading wave for both solutions is almost the same. For example, the amplitude of the Euler leading wave at $t=200$ is $A=0.06372$ while the amplitude of the Serre leading wave at $t=200$ is $A=0.06356$. Although the leading waves have almost the same amplitudes, the phase speeds are slightly different. The difference in phase speeds is demonstrated in Figure 16.

After verifying that the Serre system has dispersive shock waves that are comparable with the full Euler equations, we examine the interactions of simple DSWs starting with the head-on collision. For the head-on collision we again consider two initial waveforms similar to (27) but translated as is shown in Figure 17(a). These step functions generate two counterpropagating waves that begin to interact at approximately $t=27$. The collision is inelastic. After the collision there are two DSWs propagating in different directions on the trailing edge of the DSWs. 

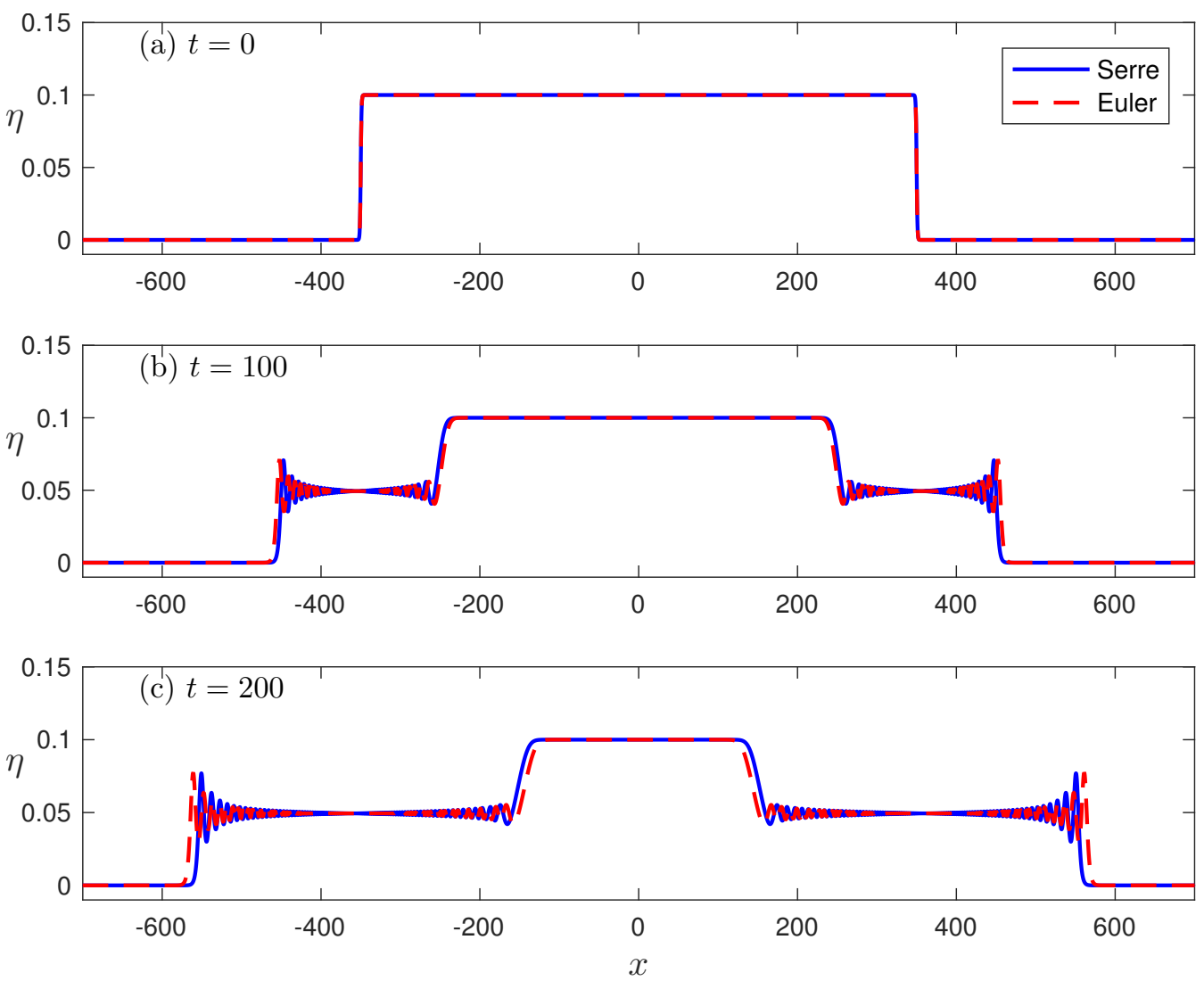

Figure 16: The dam break problem. 

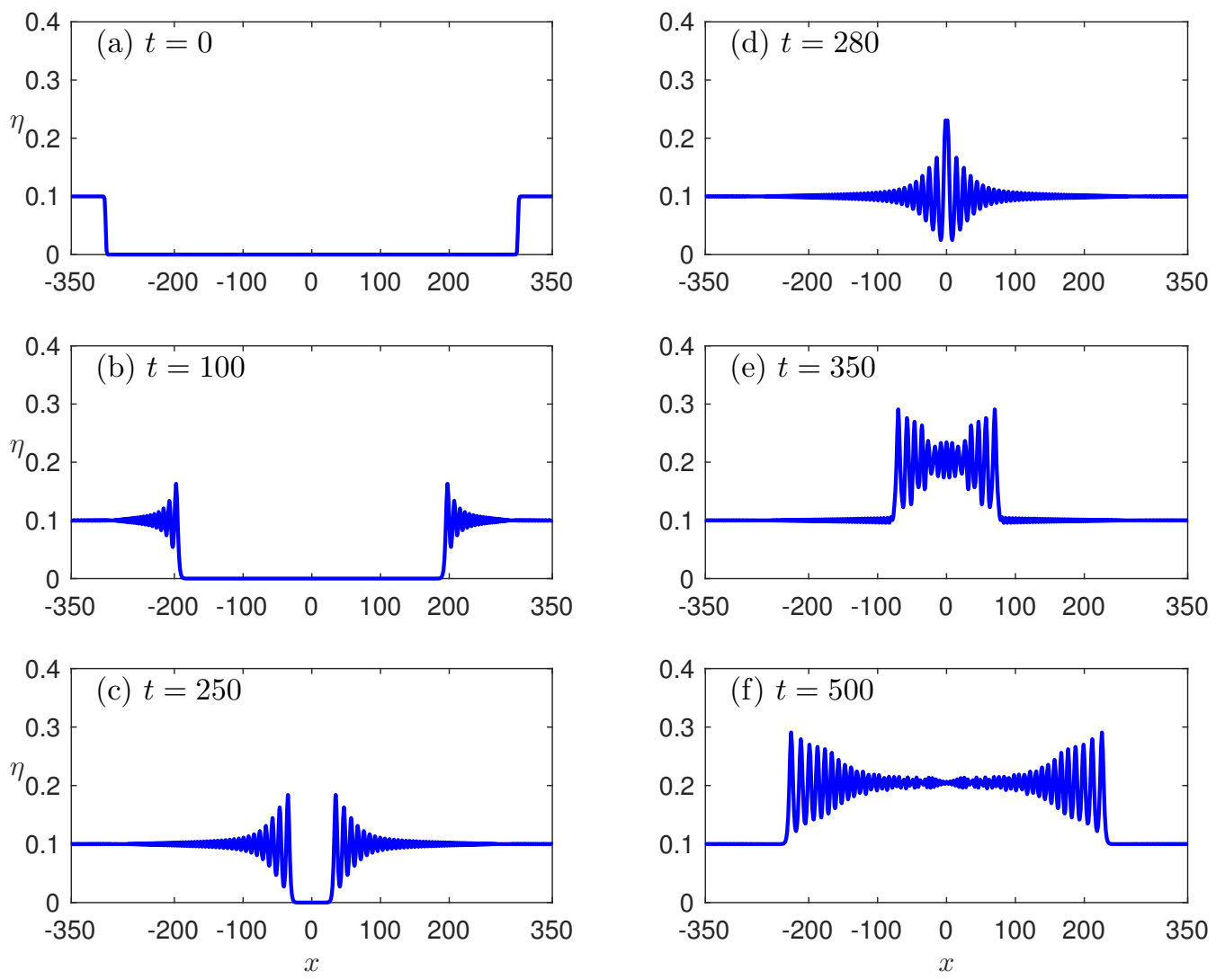

Figure 17: Head-on collision of two simple DSWs. 
We now consider overtaking collisions of DSWs. For this situation we consider double-step initial conditions as is shown in Figure 18. The first step has amplitude 0.1 while the shorter step has amplitude 0.05. This initial condition generate two DSWs that propagate to the right. Because shorter DSWs propagate with smaller phase speeds than taller DSWs, the taller DSW approaches the shorter one and they interact. The interaction is so strong that the symmetry of the leading wave of both DSWs is destroyed. The two waves appear to merge and propagate as one single-phase DSW. Similar behavior has been observed in NLS-type and KdV-type equations $[32,33]$. Finally, we mention that the solutions shown in Figures 17 and 18 are magnifications of the actual solutions. The rest of the solution, not shown in these figures, consists of dispersive rarefaction waves that we do not study in this paper. For more information see [9].

\section{Acknowledgments}

D. Mitsotakis thanks Professor Boaz Ilan for suggestions, comments, and stimulating discussions related to dispersive waves. The authors acknowledge the invaluable help of Professor Paul Milewski for discussions related to the numerical schemes for the Euler equations and Professor Didier Clamond for discussions on pseudo-spectral methods. J. Carter was supported by the National Science Foundation under grant number DMS-1107476. D. Mitsotakis was supported by the Marsden Fund administered by the Royal Society of New Zealand.

\section{References}

[1] F. Serre, Contribution à l'étude des écoulements permanents et variables dans les canaux, La Houille blanche 8 (1953) 830-872.

[2] C. H. Su, C. S. Gardner, KdV equation and generalizations. Part III. Derivation of Korteweg-de Vries equation and Burgers equation, J. Math. Phys. 10 (1969) 536-539.

[3] A. E. Green, P. M. Naghdi, A derivation of equations for wave propagation in water of variable depth, J. Fluid Mech. 78 (1976) 237-246.

[4] E. Barthélémy, Nonlinear shallow water theories for coastal waves, Surveys in Geophysics 25 (2004) 315-337. 

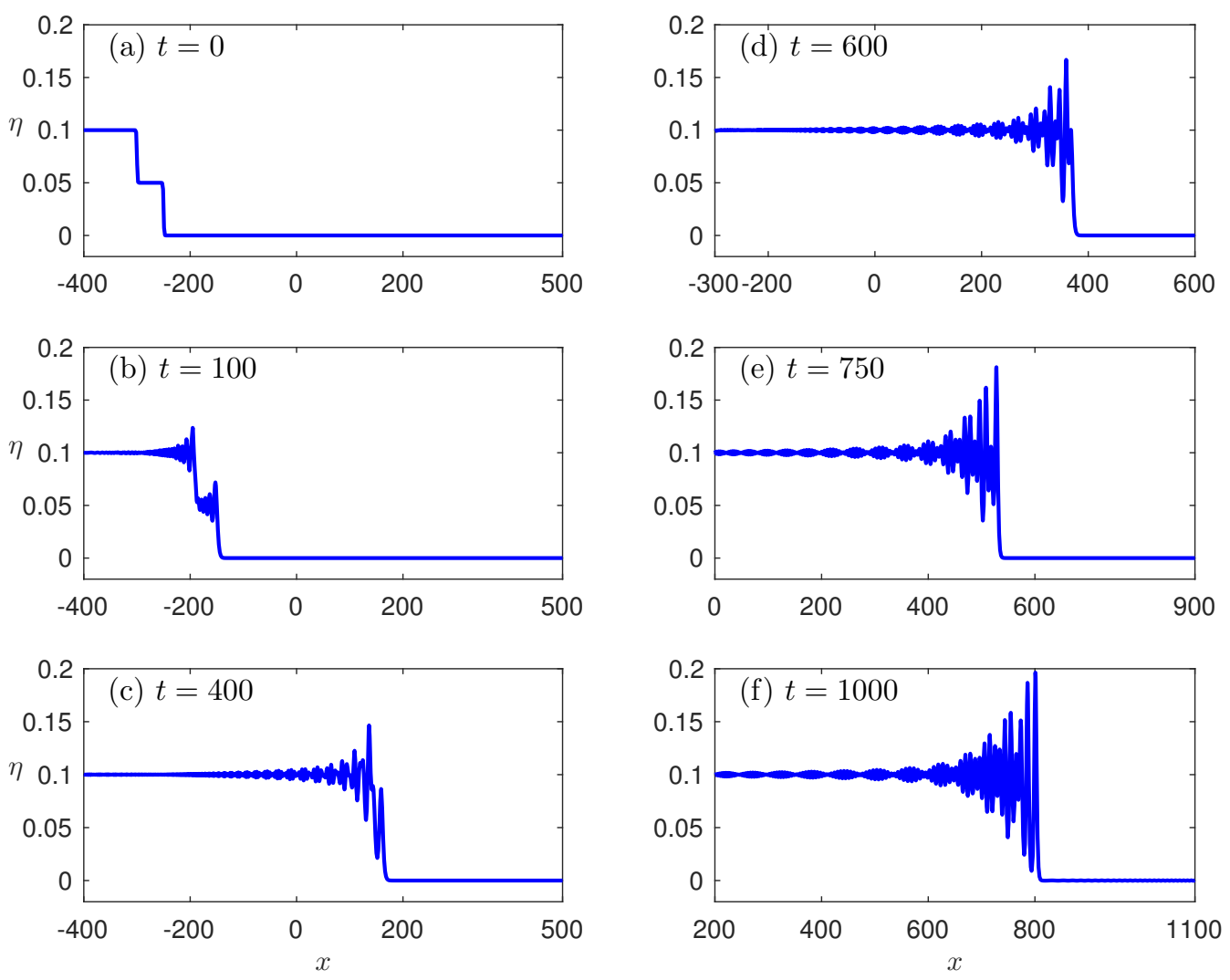

Figure 18: Overtaking interaction of two DSWs. 
[5] M. Lavrentiev, On the theory of long waves, Akad. Nauk. Ukrain. R. S. R., Zbornik Prac. Inst. Mat. 8 (1947) 13-69.

[6] T. B. Benjamin, J. L. Bona, D. K. Bose, Solitary-wave solutions of nonlinear problems, Phil. Trans. R. Soc. Lond. A 331 (1990) 195-244.

[7] J. L. Bona, Y. A. Li, Decay and analyticity of solitary waves, J. Math. Pures Appl. 76 (1997) 377-430.

[8] E. Hairer, S. P. Nø rsett, G. Wanner, Solving ordinary differential equations: Nonstiff problems, Springer, 2009.

[9] D. Mitsotakis, B. Ilan, D. Dutykh, On the Galerkin/FiniteElement Method for the Serre Equations, J. Sci. Comput. In Press. doi:10.1007/s10915-014-9823-3.

URL http://link.springer.com/10.1007/s10915-014-9823-3

[10] D. Dutykh, D. Clamond, P. Milewski, D. Mitsotakis, Finite volume and pseudo-spectral schemes for the fully nonlinear 1D Serre equations, European Journal of Applied Mathematics 24 (05) (2013) 761787. arXiv:1104.4456, doi:10.1017/S0956792513000168.

URL http://hal .archives-ouvertes.fr/hal-00587994/

[11] J. Fenton, A ninth-order solution for the solitary wave, J. Fluid Mech 53(2) (1972) 257-271.

[12] V. I. Petviashvili, Equation of an extraordinary soliton, Sov. J. Plasma Phys. 2(3) (1976) 469-472.

[13] D. Clamond, D. Dutykh, Fast accurate computation of the fully nonlinear solitary surface gravity waves, Comput. \& Fluids 84 (2013) 35-38. doi:10.1016/j.compfluid.2013.05.010.

URL http://linkinghub.elsevier.com/retrieve/pii/S0045793013001965

[14] D. Dutykh, D. Clamond, Efficient computation of steady solitary gravity waves, Wave Motion 51 (1) (2014) 86-99. doi:10.1016/j.wavemoti.2013.06.007.

URL http://linkinghub.elsevier.com/retrieve/pii/S0165212513001169

[15] L. V. Ovsyannikov, To the shallow water theory foundation, Arch. Mech. 26 (1974) 407-422. 
[16] A. I. Dyachenko, V. E. Zakharov, E. A. Kuznetsov, Nonlinear dynamics of the free surface of an ideal fluid, Plasma Physics Reports 22 (10) (1996) 829-840.

[17] Y. A. Li, J. M. Hyman, W. Choi, A Numerical Study of the Exact Evolution Equations for Surface Waves in Water of Finite Depth, Stud. Appl. Maths. 113 (2004) 303-324.

[18] J. L. Bona, M. Chen, J.-C. Saut, Boussinesq equations and other systems for small-amplitude long waves in nonlinear dispersive media. I: Derivation and linear theory, Journal of Nonlinear Science 12 (2002) 283-318.

[19] A. Duran, D. Dutykh, D. Mitsotakis, On the Gallilean invariance of some nonlinear dispersive wave equations, Stud. Appl. Math. Accepted.

[20] S. M. Mirie, C. H. Su, Collision between two solitary waves. Part 2. A numerical study, J. Fluid Mech. 115 (1982) 475-492.

[21] C. H. Su, R. M. Mirie, On head-on collisions between two solitary waves, J. Fluid Mech. 98 (1980) 509-525.

[22] W. Choi, R. Camassa, Exact Evolution Equations for Surface Waves, J. Eng. Mech. 125 (7) (1999) 756. doi:10.1061/(ASCE)07339399(1999)125:7(756).

[23] W. Craig, P. Guyenne, J. Hammack, D. Henderson, C. Sulem, Solitary water wave interactions, Phys. Fluids 18(5) (2006) 57106. doi:10.1063/1.2205916.

[24] T. Marchant, N. F. Smyth, Soliton interaction for the extended Kortewed-de Vries equation, IMA Journal of Applied Mathematics 56 (1996) 157-176.

[25] D. Dutykh, T. Katsaounis, D. Mitsotakis, Finite volume schemes for dispersive wave propagation and runup, J. Comput. Phys 230 (8) (2011) 3035-3061. doi:10.1016/j.jcp.2011.01.003.

URL http://linkinghub. elsevier.com/retrieve/pii/S0021999111000118

[26] G. A. El, R. H. J. Grimshaw, N. F. Smyth, Unsteady undular bores in fully nonlinear shallow-water theory, Phys. Fluids 18 (2006) 27104. 
[27] P. D. Lax, C. D. Levermore, The small dispersion limit of the KdV equations: III, Commun. Pure Appl. Math. XXXVI (1983) 809-830.

[28] Y. Brenier, D. Levy, Dissipative behavior of some fully nonlinear KdV-type of equations, Physica D 137 (3-4) (2000) 277-294. doi:10.1016/S0167-2789(99)00190-6.

[29] J. D. Carter, R. Cienfuegos, The kinematics and stability of solitary and cnoidal wave solutions of the Serre equations, Eur. J. Mech. B/Fluids 30 (2011) 259-268.

[30] B. Deconinck, J. N. Kutz, Computing spectra of linear operators using the Floquet-Fourier-Hill method, J. Comp. Phys. 219 (2006) 296-321.

[31] V. Ruban, The Fermi-Pasta-Ulam recurrence and related phenomena for 1D shallow-water waves in a finite basin, JETP 114 (2012) 343-353.

[32] M. A. Hoefer, M. J. Ablowitz, Interactions of dispersive shock waves, Phys. D 236 (1) (2007) 44-64. doi:10.1016/j.physd.2007.07.017. URL http://linkinghub.elsevier.com/retrieve/pii/S0167278907002412

[33] M. J. Ablowitz, D. E. Baldwin, Interactions and asymptotics of dispersive shock waves - Korteweg-de Vries equation, Phys. Lett. A 377 (7) (2013) 555-559. doi:10.1016/j.physleta.2012.12.040.

URL http://linkinghub.elsevier.com/retrieve/pii/S037596011300011X 